\title{
Webometric Analysis of Linkages between Environment Related Researchers and Institutes in Sri Lanka
}

\author{
Ambegala R.R.*, Amarasekara H.S. \\ Department of Forestry \& Environmental Science, University of Sri Jayewardenepura, Sri Lanka \\ *rajantha.rc@gmail.com
}

\begin{abstract}
Every scientist, research Institute and all research publications are connected to one another by means of affiliations, co-authorships and citations. The objective of the present study was to visualize linkages among researchers and institutes in environment related publications in Sri Lanka, aiming to identify special patterns and trends. This Webometric and Bibliometric study had three main sections: co-citation analysis, co-authorship analysis, and hyperlink analysis. In addition, an author keyword analysis and a co-citation analysis was conducted to identify different areas within the environmental science field and their relative popularity. The analysis was conducted using data gathered from the abstract and citation database "Scopus", which were published during the 10-year time period from 2006 to 2015.VOSviever was used to generate maps. Gathered data revealed that a total of 10,367 authors were involved in environment related publications. It has been observed that even though the study was conducted using Sri Lankan publications, collaboration between foreign and domestic scientists was higher than Sri Lankan scientists themselves. From subject related linkages within environment related publications, the study revealed 24 different subject areas of interest among scientists based on author keyword analysis and co-citation analysis. Out of that $13.3 \%$ and $11.9 \%$ of the publications are related to 'Ecology' and 'Agriculture' respectively. It was found that 45 institutes were involved in environment related publications. Analysis revealed that $42.0 \%$ of total citation links (Citation Analysis) and 30.9\% of total coauthorship links (Co-authorship Analysis) belong to the University of Peradeniya. Citation analysis of authors revealed 3,116 authors $(30.1 \%)$ with at least one citation link, and 7,251 $(69.9 \%)$ authors with zero citation links. Highest (10.1\%) non-domestic citation links were with United States scientists. Followed by India (6.5\%) and Australia (6.3\%).Hyperlink analysis revealed a low connectivity between institute web domains with only 11 out of 45 domains that contain more than 10 outgoing 'url citations' to one another. It was evident that the research output in subject areas such as Wildlife Management, Climate Change and Waste Management are much lower in comparison. These institutes and researchers need to keep a much higher web activity and connectivity in order to obtain a better online visibility.
\end{abstract}

Keywords: Webometrics, Bibiliometrics, Co-citation, Co-authorship, Hyperlink analysis 